\title{
Wireless Sensor Network and Internet of Things in Precision Agriculture
}

\author{
Farzad Kiani, Amir Seyyedabbasi \\ Computer Engineering Dept., Engineering and Natural Sciences Faculty, \\ Istanbul Sabahattin Zaim University, Istanbul, Turkey
}

\begin{abstract}
Internet of Things is one of the most popular subjects nowadays where sensors and smart devices facilitate the provision of information and communication. In IoT, one of the main concepts is wireless sensor networks in which data is collected from all the sensors in a network characterized by low power consumption and a wide range of communication. In this study, an architecture to monitor soil moisture, temperature and humidity on small farms is provided. The main motivation for this study is to decrease water consumption whilst increasing productivity on small agricultural farms and precisions on them. This motivation is further propelled by the fact that agriculture is the backbone of some towns and most villages in most of the countries. Furthermore, some countries depend on farming as the main source of income. Putting the above-mentioned factors into consideration, the farm is divided into regions; the proposed system monitors soil moisture, humidity and temperature in the respective regions using wireless sensor networks, internet of things and sends a report to the end user. The report contains, as part of the information, a 10-day weather forecast. We believe that with the above information, the end user (farmer) can more efficiently schedule farm cultivation, harvesting, irrigation, and fertilization.
\end{abstract}

Keywords-Wireless sensor network; internet of things; smart agriculture applications; precision agriculture

\section{INTRODUCTION}

Owing to the increment in population and corresponding decrement in rainfall amount, there is a substantial scarcity of food and water - which are the most basic needs of life. Hence, the importance of precision agriculture [1] has become more pronounced leading to a plethora of researchers being conducted in the field over the recent decades. In most countries, the family economy depends on agriculture, thus, principled and productive agriculture is of paramount importance to them. Unfortunately, in the most countries and regions where farming is done on small farms, primitive methods are still widely being used while in developed countries; statistics show that the use of modern agriculture is on the rise. Modern agriculture can be expressed with this view; reduce agricultural costs and increase productivity.

The main target of this study is small farms, in other words, farms capable of planting several types of products, such as vegetables and fruits in a small area. For instance, consider the cultivation of eggplant, parsley, pepper or tomato on a farm, each of these needs a different irrigation method and scheduling. Thus, the main goal of this study is increasing production with minimal costs and, ultimately, increase net revenue. Technology supported agriculture with concepts of wireless sensor networks (WSN) and the Internet of Things (IoT) can help to gradually shift from primitive agricultural practices. Studies on industrial agriculture ranging from climate information, soil information to intelligent irrigation was based on data collection performed by wireless sensors. The wireless system enhances crop productivity and convenience [2]. WSNs collect information from different sensors in large and small networks so end users can get and process data. These networks can be used in the monitoring of people health, weather conditions, control traffic, and air pollution.

IoT is an environment where objects, animals or people are equipped with unique identifiers capable of data transmission over the internet without the need for human or computer interactions [3]. In this paper, we effort to simulate IOT concept using different sensors. The information collected by the sensors is sent to the server with RASPBERRY PI 3 and WI-FI module that can be understood in a graphical environment (GUI). As days go by, the number of applications in this field increases and many researchers have been focusing on a new idea based on IoT technology concept. One of the popular research areas is agriculture and smart farms. However, they are not comprehensive methods. The most researches are suffered from compatibility and connection between heterogeneous devices [4]. Currently, the farmer can collect data as temperature, air humidity, soil humidity, volumetric water content unit (VWC) and gravimetric water content (GWC) from the different regions of the farmed area thanks to this technology. After collecting the required data, they can be analyzed and give flexible plans to its users in order to different intentions such as cultivation, harvest, irrigation, fertilization. It is also important to note that the collected data by digital sensors must be understandable to the end user.

In our proposed system, we collect different data via sensor nodes. In addition, we use some current information such as weather conditions because analysis of the system that takes external factors into its own parameters is more reliable.

The remainder of the paper is organized as follows. Section 2 is about related works of precision agriculture, Section 3 describes our proposed system architecture and abilities. Finally, Section 4 presents conclusions of the workstudy and future works. 


\section{RELATED WORKS}

In this section, we focus on how to use the IoT and smart technologies in the agriculture-based applications.

In [2] has proposed a smart wireless sensor network so the authors design an architecture to data collection from nodes in an agriculture environment. They analyze collected data and display their results to its end users. In [5] has presented a method based on wireless sensor networks in potato farming that it monitors and understands individual crop and requirements. Therefore, the farmers can potentially identify the various fertilizers, irrigation, and other requirements. The authors propose an irrigation management model to estimate agricultural parameters using mathematical calculations and intelligent humidity sensors. Devices used for monitoring are laptop computers or PDA. In [6] has introduced a smart system based on wireless sensor network in a red bayberry greenhouse using soil moisture and temperature sensors. This system can collect the temperature, humidity, illumination, and voltage of the greenhouse.

GPRS gateway has been used for transmitting data to monitor the system. The energy of sensors is provided by solar and storage batteries. In [7] has proposed a novel platform to establish energy efficient wireless sensor network in a sugar farm. It provides the energy efficiency by the solar system. In [8] has proposed a method to make agriculture smart using automation and IoT technologies. In this paper, the authors use ZigBee models, camera and actuators to handle smart irrigation on accurate real-time field data. In [9] has focused on automated irrigation using the wireless moisture sensor network and IoT technology in order to smart precision agriculture system. Irrigation by schedule is to supply water to the plant at specific times and automated irrigation by feedback based on temperature, humidity and moisture sensors. Two irrigation methods are compared to the second method has better efficiency in water consumption.

As seen in many studies in the literature, wireless sensor networks are a structure that works nested with IoT technology. Therefore, let us talk a bit about this network.

\section{A. Wireless Sensor Networks}

The wireless sensor networks (WSNs) consist of multiple many sensor nodes in a wireless communication-based environment. The sensor node is to detect physical phenomena such as temperature, humidity, and moisture with limited energy and memory [10]. Many current researchers are focused on these limitations by various methods as different routing protocols [11], [14], intelligent based approaches [12], [13] etc. They have many applications area in various environments such as military, medicine, education, agriculture, monitoring systems etc. It is expected that it will be more workspaces by emerging the IoT concept. Fig. 1 shows a sample architecture of a sensor node.

The main components of a sensor node are power unit, computing unit, sensing unit and Communication unit.

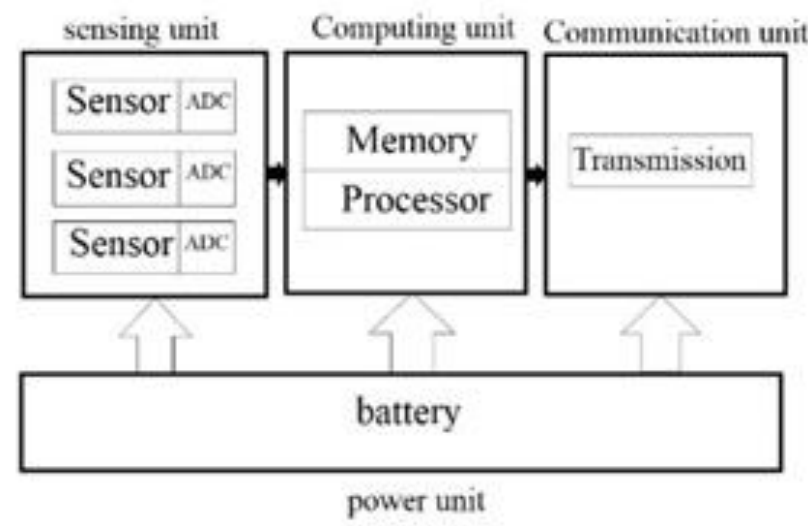

Fig. 1. Node architecture.

The nodes sense a physical event of the phenomenon and convert them to digital signals by sensing unit. Then they handle collected data and process them by computing unit. This unit is consist of processing and memory subunits. They can store necessary data in their storage. In addition, they support necessary their operations from a few hours to months or years via power unit. The sensor nodes are also able to communicate together or server/base station as centralized or decentralized architectures based on various topologies such as mesh, star etc. This is realized via the communication unit. The sensor nodes can communicate together in the point-to-point or multi-hop models [14].

These are the deepest differences with the classic sensor nodes. These nodes furthermore widely deployed in $1 \mathrm{~m}$ up to 100meters area, low communication bandwidth, limited memory and computation power.

The characteristics of wireless sensor nodes are ease of use, scalability to the large scale of deployment, the mobility of nodes and resilience. Since these networks are interested in information regarding the physical phenomenon instead of information from a single sensor, the failure of a single node should not affect the overall operation of the network. Nevertheless, the fault tolerances is an important design factor in the networks [15]. Need to be aware of other factor is cost. The most basic features of these networks are cheap. Therefore, they are almost used as low-cost sensor nodes. Researchers are introduced new sensor devices with different software and hardware properties. In this paper, we use our designed sensor node. The other factor is energy and network lifetime. The sensor nodes have low battery level (4 Joule or less). Various methods such as optimized routing protocols, topology control, and power management protocols are followed to achieve this aim.

\section{B. Internet of Things}

Internet of Things (IoT) is one of the new topics that has been discussed recently that usage areas are growing rapidly. They are consist of many nodes that are equipped with internet output. The existing technologies such as ad-hoc systems, 
pervasive and embedded systems, wearable technology and machine learning techniques are founded new concept by emerging of IoT. One of the most important requirements in this technology is connection to internet problem [16]. Internet one of the inseparable things in our life that can reduce cost and time. Imagine yourself doing an internet search for your watch you lost somewhere in your house. Therefore, this is the main vision of IoT, an environment where things are able to talk and their data can be processed to perform desired tasks through machine learning techniques [6], [13]. The IoT is built around wireless radio waves that allow to different devices to communicate with each other through the Internet. This platform includes some standards such as Wi-Fi, low-power Bluetooth, NFC, RFID, and so on. Physical object in IoT collects and process data that it can receive it from the environment and/or other objects [17]. These objects are embedded with sensors, actuators, internet output and network connectivity. Truth, not all the devices need to be the connected internet. This property can be increased the cost of the system. In this case, a system designer can use the WSN structure in order to reach to a smart application.

Environmental and personal health monitoring, monitoring and control of industrial processes including agriculture, smart spaces, and smart cities are just some of the examples of IoT applications [3]. According to Fig. 2, it is estimated that by 2025 approximately 75.44 billion devices will be connected to the internet [18]. One of the IoT trends is in agriculture. In agriculture applications based on IoT, the objects communicate together to provide useful information from the farm or greenhouse. In these types of systems, some actual devices do some required operations such as irrigation and prune. In other words, the internet and physical agents have been effective in reducing the human factor involvement, to increase productivity, as well as reduce costs.

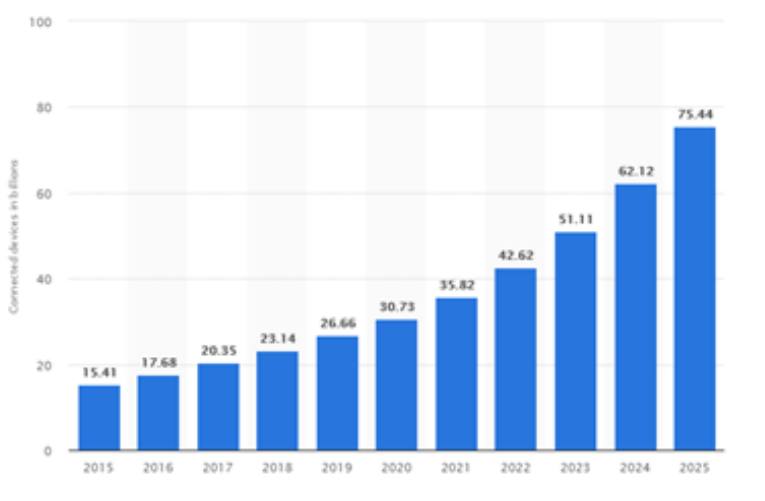

Fig. 2. Internet of Things (IoT) connected devices.

\section{PROPOSED SYSTEMS}

In this section, the proposed system architecture is introduced. The used sensor types in the system are humidity, temperatures, and soil moisture. In this architecture, the sensorbased devices are deployed in the environment to sense and data collection. The related system has more benefit for farmers so he/she can manage his/her time, energy and costs. Data will be collected by system nodes and it transmits to server finally via the cross from probable other nodes and gateways. The all collected data in server side must be analyzed and be presented to the users in a user-friendly platform. SIXFAB Company and IZU-WSN Research Lab. designed a custom board that is called as KIANI sensor nodes (Fig. 3). This node is used in this case study.

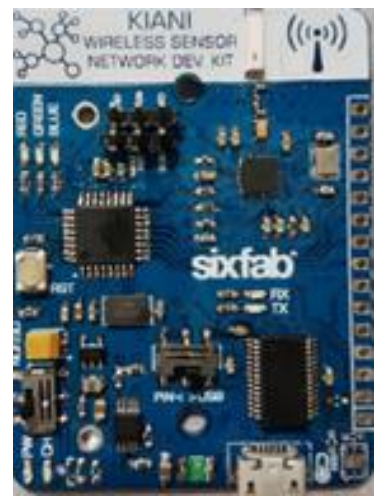

Fig. 3. Our jointly produced custom sensor node.

The sensing unit of the node has SparkFun Soil Moisture Sensor. Furthermore, the board has temperature and humidity sensors that sense different air humidity and temperature. In communication unit, our board transceiver is based on Texas instruments cc1101 Low-Power Sub-1GHz RF Transceiver Computing unit equips with Arduino Nano, ATmega328P used in computing unit. In power unit, we used 1200mah 3.7v Li-ion rechargeable battery. In Second part of the architecture, we use RASPBERRY PI 3 as a gateway to send collected data from sensors to sever for processing and presentation information that users need to it. At last, related information such as temperature, humidity, soil moisture and future air conditions are shown as an application in GUI that can be available in the related website and mobile application. Fig. 4 shows a sample model of the system and node in the agricultural land.

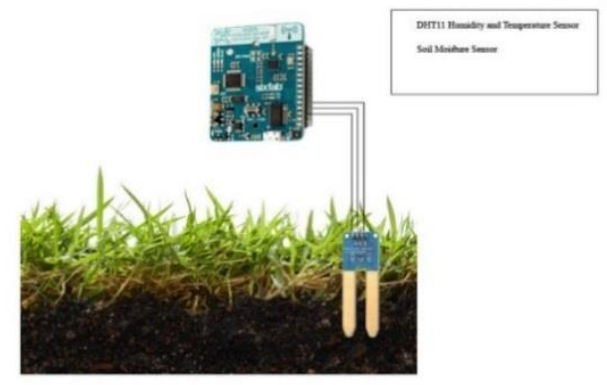

Fig. 4. Sensors based custom nodes in the farm.

Today, the water issue is one of the important problems in our life. We need to avoid the litter and we must use this source correctly, especially in agriculture lands.

On the other hand, the irrigation system has traditionally used in farms and water comes to the point of water entry into the farms. In result, the water has come to the total agricultural land over a given period. To cover the total farm surface, it also depends on the surface of the earth and type of soil. Traditionally, created waterways in the farms could divide the entrance water at least equal. In our proposed system, we 
consider a farm with $100 * 100$ meters that farmers divided this area into four equal regions as is shown in Fig. 5. Then, in each region, our nodes have been deployed. Data is obtained in every one hour from devices, which forwards it to the gateway. Where it is stored and then transferred to end users through API.

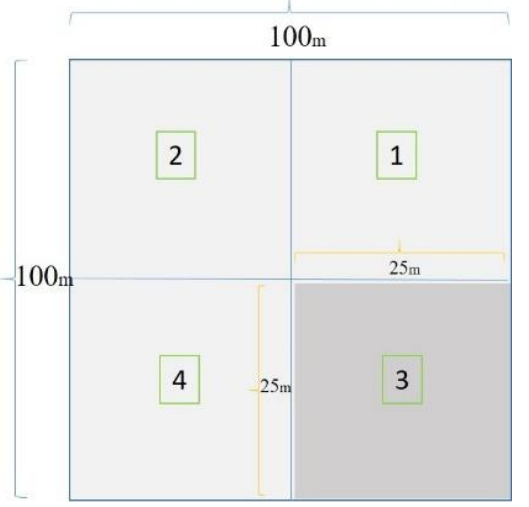

Fig. 5. The farm structure that is divided into 4 equal clusters.

After this, an application will be processed and data will be shown to end user. The working mechanism of the proposed system is presented in Fig. 6. It shows related information in every region in one-hour periodic periods, continuously. The results of the system are presented to end user via a website or mobile application so is shown in Fig. 7. The proposed application uses the current some information as weather condition besides the information that receives from our nodes.

The end user with this comprehensive information will be prepared fields of farms for planting, harvesting, fertilizing and irritating. This general view of the farm, the farmer will be aware of the accurate understanding of land changes, which previously was traditionally used and experimental knowledge was provided. In such systems, technology will provide useful information at the lowest cost. Launching the system may be costly for the farmer for the first time, but over time, with the increase in productivity, this cost will be offset. The work is done automatically and farmer monitors self-land makes some decisions and applies them through automatic devices that are in the farm or near to our region.

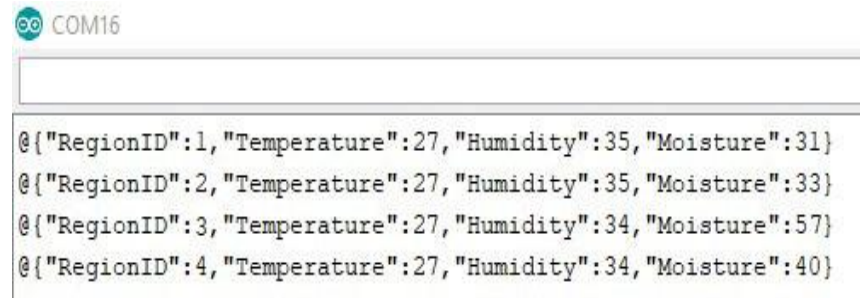

Fig. 6. Data comes from Arduino.

This system provides a great convenience to farmers and they can have more and more efficient products, efficient use of water and management of energy, time and unnecessary classic costs. All of them would be one of the reasons why farmers would be welcome. The implemented GUI has a userfriendly interface so the users can benefit from all means. In this system, we tried to have a simple and understandable graphic user interface. The system will update the information hourly, as well as provide a history of the information from every region.

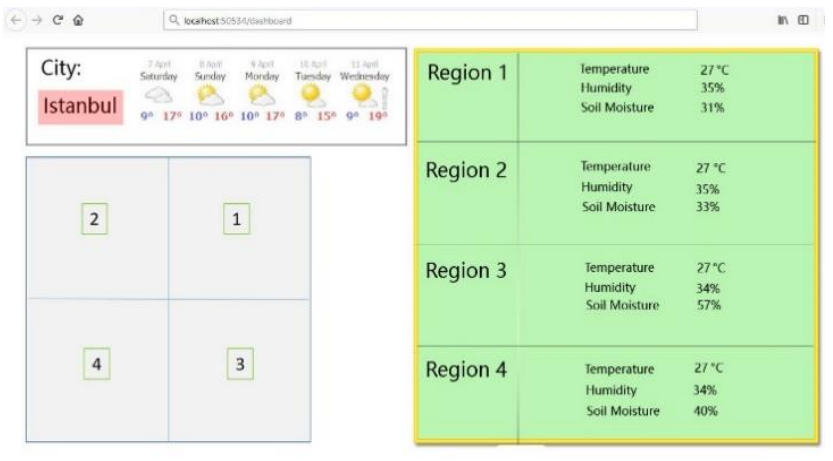

Fig. 7. GUI snapshot.

\section{RESUltS AND EVALUATION}

IoT's importance is increasing day by day in our lives. There are a variety of applications in this respect. Because these methods are inspired by WSN technology, they can also be used to differentiate between different algorithms and software methods. They can be more usable in various applications so one of them is agriculture area. In today's world, people are beginning to make use of intelligent devices and system thus; they have a smart assistant thanks these systems. In this paper, an architecture has been proposed so it helps to farmers to manage the irrigation time of their agriculture correctly. The result of it is shown it is efficient in the resource consumption. The resulting of the system that is an application can be of great benefit to its users. The user can directly divide own land into as many regions as desired in this application. Therefore, the users will provide saving in their time and water. In addition, this application is reliable because it provides weather information instantaneously from the central stations.

\section{CONCLUSION AND FUTURE WORKS}

In general, water issue and irrigation methods play an important role in efficient water using and increase productivity. So, water consumption reduction that helps farmers economic at the small farms. Furthermore, farmer's information about weather conditions of next days can help to make decisions that are more accurate. In this project, we used the IoT and WSN enables to achieve this goal. In addition to, fertilization, harvesting and cultivation are important as irrigation too. Therefore, with this method, the farmer can schedule his/her next upcoming activities. About future work, we will use a reinforcement learning based system in data collection and processing phases on the farms to give suggestions that are more useful to farmers about normal activities in farms. In addition, some data can be collected from self-farmers.

As future works can design different hybrid architectures for various IoT based applications. It can be realized by 
software and hardware-based approaches. In addition, the machine learning-based methods such as reinforcement learning, game theory, fuzzy logic and neural networks can be suggested. On the other hand, the energy efficiency issue can be investigated from different perspectives such as routing and communication between devices based on shortest path finding methods.

\section{ACKNOWLEDGMENT}

This project has been achieved in Wireless Sensor Networks and IoT Laboratory in Istanbul Sabahattin Zaim University (IZU). Many thanks to our colleagues at this laboratory and SixFab Co. that help us in this project.

\section{REFERENCES}

[1] Z. Naiqian, M. Wang and N. Wang. "Precision agriculture-a worldwide overview." Computers and Electronics in Agriculture 36.2-3, pp. 113132, 2002.

[2] G. Mendez, M.A. Yunus and S.C. Mukhopadhyay. "A WiFi-based smart wireless sensor network for monitoring an agricultural environment". IEEE Conference in Instrumentation and Measurement Technology Conference (I2MTC), pp. 2640-2645, 2012.

[3] A. Gluhak, S. Krco, M. Nati. "A survey of facilities for the experimental internet of things research". IEEE Communications Magazine, 49(11), pp.58-67, 2011.

[4] T. Ojha, S. Misra and N. S. Raghuwanshi "Wireless sensor networks for agriculture: The state-of-the-art in practice and future challenges". Computers and Electronics in Agriculture, 118, pp.66-84, 2015.

[5] K. Shinghal and S. Neelam. "Wireless Sensor Networks in Agriculture: For Potato Farming". Available at SSRN: https://ssrn.com/abstract=3041375, 2017.

[6] X. Jianfa. "An environment monitoring system for precise agriculture based on wireless sensor networks". IEEE International Conference Mobile Ad-hoc and Sensor Networks (MSN), pp. 28-35, 2011.
[7] P. Milind. "H2E2: A hybrid, hexagonal \& energy efficient WSN green platform for precision agriculture". IEEE 12th International Conference on Hybrid Intelligent Systems (HIS), pp.155-160, 2012.

[8] G. Nikesh, R.S. Kawitkar. "Smart Agriculture Using IoT and WSN Based Modern Technologies". International Journal of Innovative Research in Computer and Communication Engineering, 4(6), pp. 12070-12076, 2016.

[9] M. Ibrahim, M. Rawidean, M. Kassim and A.N. Harun. "IoT in precision agriculture applications using wireless moisture sensor network". IEEE Conference on Open Systems, pp.24-29, 2016.

[10] S. Lachure, A. Bhagat and J. Lachure, J. "Review on precision agriculture using wireless sensor network". International Journal of Applied Engineering Research, 10(20), pp.16560-16565, 2015.

[11] F. Kiani, E. Amiri, M. Zamani, T. Khodadadi, and A. Abdul Manaf, "Efficient intelligent energy routing protocol in wireless sensor networks", International Journal of Distributed Sensor Networks, 2015, pp.1-13.

[12] F. Kiani, "AR-RBFS: Aware Routing Protocol based on Recursive BestFirst Search Algorithm for Wireless Sensor Networks", Journal of Sensors, 2016, pp.1-11.

[13] F. Kiani, "Reinforcement Learning based Routing Protocol for Wireless Body Sensor Networks". The 7th IEEE International Symposium on Cloud and Service Computing, pp.71-78, 2017.

[14] K. Sohraby, M. Daniel and Z. Taieb. "Wireless sensor networks: technology, protocols, and applications". John Wiley \& Sons Book, 2007.

[15] F. Akyildiz and M. C. Vuran. "Wireless sensor networks". John Wiley \& Sons Book, 2010.

[16] M. Stoces, J. Vanek, J. Masner and J. Pavlík. "Internet of things (IoT) in agriculture-selected aspects". Agris on-line Papers in Economics and Informatics, 8(1), pp.83-89, 2016.

[17] L. Atzori, A. Iera and G. Morabito, "The Internet of Things: A survey," Computer Network, 54(15), pp. 2787-2805, 2010.

[18] S. Yerpude, T. K. Singhal. "Impact of Internet of Things (IoT) Data on Demand Forecasting”. Indian Journal of Science and Technology, 10(5), $1-5,2017$. 